\title{
The Case of Child Abuse and Neglect: Identification of Non-accidental Injuries by Medical Professionals at the Emergency and Trauma Department
}

Submitted: 05-11-2018

Accepted: 06-12-2018

\author{
Anne Noor Sri Juwaneeta Jamaludin', Jennifer Oxley ${ }^{2,3}$, \\ Muhammad Ashraff Ahmad ${ }^{4}$, Quek Kia Fatt ${ }^{2}$ \\ ${ }^{1}$ Perdana University-Royal College of Surgeons in Ireland, Selangor, \\ MALAYSIA \\ ${ }^{2}$ Monash University Malaysia, Selangor, MALAYSIA \\ ${ }^{3}$ Monash University Australia, Clayton, Victoria, AUSTRALIA \\ ${ }^{4}$ University Malaya, Kuala Lumpur, MALAYSIA
}

To cite this article: Jamaludin ANSJ, Oxley J, Ahmad MA, Quek KF. The case of child abuse and neglect: identification of non-accidental injuries by medical professionals at the emergency and trauma department. Education in Medicine Journal. 2018;10(4):93-106. https://doi.org/10.21315/ eimj2018.10.4.9

To link to this article: https://doi.org/10.21315/eimj2018.10.4.9

\section{ABSTRACT}

It is well established that accidental and non-accidental injuries/child abuse and neglect (CAN) are a major public health problem globally. Not only do they affect individuals, injuries affect families, the community, government and internationally as well. Injuries span throughout childhood and into adulthood. Purpose of this study is to identify what are the difficulties and challenges in identifying and reporting CAN cases at the emergency department (ED) in Malaysian hospitals. Thirty in-depth interviews were conducted at the ED in three major hospitals in the Klang Valley, Malaysia which consists of specialists, medical officers, nurses and medical assistants. The study found that there is a significant gap identified in medical professionals' knowledge and skills related to understanding, identifying and detecting CAN, particularly among medical officers who are in charge of diagnosis and reports in the hospital. Those who had previous experience or encounters in dealing with CAN cases would have higher suspicion index compared to those who have not. Other medical professionals such as nurses and medical assistants although do not have the mandate to diagnose or report of CAN cases, needed to have the basic knowledge and awareness and play more active role in their respective settings.

Keywords: Child abuse and neglect, Non-accidental injuries, Childhood injuries, Qualitative research Ireland, Block D, MAEPS Building, MARDI Complex, Jalan MAEPS Perdana, 43400 Serdang, Selangor, Malaysia | E-mail: annejamaludin@perdanauniversity.edu.my

\section{INTRODUCTION}

Although communicable diseases are still the primary killer of infants, World Health Organisation and UNICEF's
World Report on Child Injury Prevention (1), indicated that injuries contributed significant proportions of deaths for children aged between 5 and 14 years (27\%) and adolescents/young adults aged 15-29 years 
$(40 \%)$. Drowning and road traffic injuries are among the top ten cause of death amongst children within the age of 1 to 14 years, while road traffic injuries was the number one cause of death for adolescent age between 15 and 19 years old. Each year, injury and violence kill up to 950,000 children under 16 years of age and millions more suffer long-term consequences of non-fatal injuries (2). Comparing to other regions, UNICEF reported about $60 \%$ of road traffic injury fatality cases occurred in the South East Asian and Western Pacific Regions alone. It is estimated that over 2,000 children died everyday due to injury worldwide, while many more would sustain severe injury outcomes on a daily basis; putting a strong emphasise on the importance and significance of burden of injuries to children (1). The purpose of this study is to determine the current indicators used by medical staff to identify intent of child injury and the processes that are undertaken once potential child neglect, maltreatment or abuse is identified (i.e. police report, department of social and welfare, etc.).

\section{METHODS}

Participants were recruited from three major hospitals in the Klang Valley (Selayang Hospital, Serdang Hospital and Sg. Buloh Hospital) and included Emergency Department (ED) specialists, paediatricians, medical officers, nurses and medical assistants using purposive sampling. Participants were invited to participate and recruited specifically if they worked directly with children presenting at the hospital (mainly at the Emergency Department and Paediatric Department).

A total of 30 participants were recruited and interviewed from all three hospitals. Housemen were excluded from the study as they are trainees and would not have enough experience, knowledge and skills within the area of paediatric injuries (identification of accidental and non-accidental injuries).
Medical officers, nurses and medical assistants who have less than three years of experience working at the Emergency and Trauma Department were also excluded. The number of healthcare professionals interviewed and years of service are shown in Table 1.

Table 1: Frequencies and Percentages for Demographic characteristics of participants

\begin{tabular}{lcc}
\hline Variable & $\mathbf{N = 3 0}$ & $\%$ \\
\hline Gender & & \\
$\quad$ Male & 12 & 40.00 \\
$\quad$ Female & 18 & 60.00 \\
Ethnicity & & \\
$\quad$ Malay & 25 & 83.30 \\
$\quad$ Chinese & 2 & 6.70 \\
$\quad$ Indian & 3 & 10.00 \\
Position & & \\
$\quad$ Specialist & 5 & 16.67 \\
$\quad$ Medical Officer & 11 & 36.67 \\
$\quad$ Nurse & 10 & 33.33 \\
$\quad$ Medical Assistant & 4 & 13.33 \\
Years in service & & \\
0-5 years & 12 & 40.00 \\
6-10 years & 12 & 40.00 \\
11-15 years & 5 & 16.67 \\
16-20 years & 1 & 3.33 \\
\hline
\end{tabular}

A higher proportion of females (60\%) compared to males $(40 \%)$ were interviewed, and a range of experience levels were shown amongst the sample. In general, there was a high level of experience with participants working around eight years on average. Only two participants had worked for a minimum of two years and one for 20 years.

\section{RESULTS}

A qualitative study based on the I-change model of behaviour and suggested coding for NVivo analysis were used for this research. There were three major themes emerged, "Determinants of Awareness of Healthcare Professionals", "Determinants 
of Motivation of Healthcare Professionals" and "Determinants of Action of Healthcare Professionals". However, this article will only be focusing on the first major theme while the remaining major themes will be presented in subsequent publications.

There were four key themes emerged housed under the major theme of "Determinants of Awareness of Healthcare Professionals". The four key themes identified are "Trigger for Actions", "Knowledge", "Risk Perception" and "Awareness of Detection Tools". The first two key themes consist of several subthemes such as "Signs of Abuse" and "Sources of Signs for Abuse" emerged for the first key theme while subthemes such as "Defining Child Abuse", "Inadequate Training and Availability of Training" and Content of training" emerged for the second key theme.

\section{Major Theme 1: Determinants of Awareness of Healthcare Professionals}

\section{Key Theme 1: Trigger for actions}

Determination on how would the participant identify whether a child is suspected of being abused or not when presented at the ED (whether at first triage, second triage or in the consultation room) were enquired and the following subthemes emerged from these questions as discussed below.

\section{Subtheme 1: Signs of abuse}

Physical signs are the main indicator for most medical officers, nurses and medical assistants in this study in detecting abuse in the emergency settings as there is an absence of detection and screening tools being currently used in any of the hospitals to detect child abuse. Inconsistency between patient history with their physical signs are the most reliable indicators of abuse observed in a child who came with injuries by most medical officers. One medical officer noted:
If it's a physical abuse, uhmm... well, we'll talk about history first. History, there will be probably inconsistencies, and then there will be changes in the history as well, delayed in coming to seek treatment.

This statement was supported by another staff nurse,

When the baby was brought in, she couldn't lift her arm. From her hand to her feet, I can't remember. And then when we did the X-ray, we found a fracture, it shows that it's broken, it does not corroborate to what the parents had told us.

Presentation of injuries itself were also observed by the participants as "red flags" for abuse. For example, an atypical fracture such as spiral type of fracture, or an atypical place for abrasion such as at the posterior triangle of the neck or the inner thighs which usually happens when a child is being sodomised, or even specific pattern of injuries such as a buckle-mark, a cigarette mark, or a pinch mark were noted as "red flags" for abuse. Signs that showed intracranial bleed were also considered a "red flag" for abuse such as sudden seizures in a previously healthy child and an upper neurological sign such as hypertonia.

In identifying signs of neglect, hygiene aspect of a child is observed by the staff nurse. Severe presentation of unhygienic features and severe malnutrition are usually picked up as a sign of neglect as mentioned by one nurse mentioned on a case that caught her eye,

But when I looked at the hygiene of the child, she looked really dirty, she was like...maybe had not had a shower for a long time maybe, like dirty, a lot of dirt, like...she had a lot of scabies...I feel like the child is not being taken care properly. 
The staff nurse focused on the hygiene aspect of a child to detect neglect in the child. It should be noted that severe presentation of unhygienic features and severe malnutrition is usually picked up as a sign of neglect. Immunisation record and the state of nutrition of the child can also be used to detect neglect as highlighted by another medical officer.

Child refusal showed towards his or her perpetrator serves as an important indicator of psychological and emotional abuse noted by many interviewed healthcare workers. A nurse recalled one of the cases that she encountered,

\begin{abstract}
And when the mother came to pick up the child, the child did not want to go with her. The child only wanted the father. So something is wrong there.
\end{abstract}

However, only a few participants highlighted the fact that abuse can be psychological, neglect or emotional versus a high proportion of participants emphasising more on physical type of abuse, which typically can be seen visibly on a child upon presentation at the Emergency and Trauma Department. .

\section{Subtheme 2: Sources of signs of abuse}

Little was mentioned on importance of sign of abuse source by most healthcare worker but it was noted that the person who brought in the child to the hospital for examination was a helpful indicator of concerned source that suspected abuse was done onto the child. A teacher, a parent or a police officer who came to the emergency department with a child is some of example denoting source as illustrated by one of the nurses,

The teacher will make a report. The student didn't come to school for three days, and when the student came, he/she was wearing worn out clothes, slow in learning and with other types of injuries. The teacher suspected the child was abused by his/her parents. The teacher or the police took the child in, the police did.

This signified the important role of teachers and authorities in recognising child abuse in the community. One specialist stated that abuse cases would continue to be underreported and undetected at the clinical setting without concerned teachers, neighbours or relatives close to the child played their part in reporting.

But sometimes when there is conflict between both parents (such as fighting for child custody), they tend to manipulate the system to accuse the other party as perpetrator in order to take full custody of the child. This was mentioned by one of the specialists during the interview,
There was one case, parents are divorced, the father accuses the mother of neglecting the children. Always leaving the children at the babysitter, and only came back late at night. This is because of child custody battle of the children. This case is currently under the Department of Social and Welfare (DSW), so still under investigation. The mother of the children instead accuses the father of abusing the children. Both are very educated, mother a teacher, father a doctor.

Such situation complicates the case further when clinical examination on abused is misused for personal gain. Therefore, sources of sign abuse can still be assessed depending on case by case nature to determine the intent of the person bringing the child in for clinical examination.

\section{Key Theme 2: Knowledge}

Understanding of child abuse definition from the participants' perspective were sought out through several questions posted. Most of the participants struggled to provide full definition of child abuse and resorted to examples instead as shown below. As such 
their understanding of child abuse affects their capability in identifying children who are potentially at risk of being abused which are discussed further in the subthemes below.

\section{Subtheme 1: Defining child abuse}

Most healthcare workers were well-informed about the definition of child abuse and some were aware on the different types of child abuse such as physical abuse (bruises, fractures, etc.) mental abuse (change in attitude, verbally abused, etc.), and neglect (malnourished, unhygienic etc.). One doctor mentioned that any harm that came to a child with intention was a form of child abuse,

For me child abuse...I'd say it can be...it is actually an intentional act of intention to harm the child. That is, including negligence also.

Whereas most participants would define child abuse as physical abuse more frequently as compared to other invisible types of abuse,

Child abuse...uhmm...you can divide into two whether the mentally or the physically abused. The physically abused sometimes we can see the signs and symptoms quite clear. If he's not brought by the one who abused them. If brought by other relatives. But mentally abused is a bit challenging because history taking is a bit difficult.

There are also some whom found it hard to separate the physical abuse and neglect completely.

One medical officer illustrated this in one of the interviews,

There's a very thin line when talking about is it a true abuse, and a true neglect. Say, a child who sustained, you know some huge bruise on the cheek or on the forehead. Because this child is now nine months old, knows to roll over and was put on a sofa. Mom just turned her back momentarily and the child fell. It's a form of neglect too.

Thus, a high proportion of participants indicated that they would need more training in order to discover signs of these abuses when facing such type of cases in the hospital despite claiming to know the variety types of abuses other than physical. Some are concerned that they might be misdiagnosing a child as being neglect if based on appearance and malnutrition. As explained by one specialist,

If the child comes in looking a bit malnourished and not wearing clean clothes while the parent is very presentable, can you simply diagnose the child as being neglect? The parent would become defensive and decided to take the child out without being treatment. How severe the neglect that you can diagnose it as an abuse?

The difficulty in making diagnosis decision on neglect is not only experienced by junior medical officer whom would rely on guidance from their supervisors but also to those whom have been dealing with such cases for such a long time. Child abuse cases were not commonly found in the hospitals where this research was conducted. Some said that in a month maybe around 5\% of children came with injuries consistent with abuse. Therefore, even though these healthcare workers knew the text-book signs of child abuse, many did not have enough experience when it comes to recognising the signs, especially the non-physical signs.

Emotional and psychological abuse were often subtle and could be missed easily by the healthcare workers as indicated by some of the participants that called for higher awareness to be created to lower down probability of missed cases. This is mentioned by one medical doctor in one of the interviews, 
But when it comes to those who don't have physical signs, such as the emotionally abused, the neglected ones, uhmm I would really want to know more about it. Because, this is that...when we say that the adults are neglecting them, we as clinicians also fail them, because the symptoms and signs are very subtle. Unless you have the awareness, you can pick them up.

Many healthcare workers also based their action on their own intuition and their own experience in dealing with child abuse cases instead of consistent training. One medical officer noted,

Even sometimes there's no signs, no obvious signs of child abuse but based on suspicions we can categorise...or we can already have the...lodge a report towards... I mean, against child abuse, for child abuse.

Role of training then becomes a pivotal element to educate junior staffs on identifying signs of neglect or abuse as they are not experienced enough in recognising the signs, limiting the early opportunity of case to be reported to required authorities.

\section{Subtheme 2: Inadequate training and availability of training}

Although some training sessions were made available at these hospitals, it is not easily accessible due to low frequency of availability and long wait list to accommodate increasing number of healthcare professionals that are new within the department. This problem was highlighted by one of the participants,

There's a training, but it's a rotational basis, that they will pick uhh...during this time, they will pick three people at most first.

The reasons that this kind of situation occurred was explained by one of the specialists interviewed,
The Ministry of Health, I would say, honestly, cannot provide enough funding to send everyone for the good courses, you know, so a lot of times, again, if you are selected, if you're lucky, then you get to go, if not, you have to sacrifice your own money to go for it.

This would setback participants up to thousands of ringgits as some of the most comprehensive courses/trainings in child protection are only available overseas. Apart from lack of funding, other reasons of not going to training was given such as insufficient number of human resources in their department to take charge in their absence, and also not having an available place in the training course.

Proactive healthcare workers would resort for self-directed learning through medical textbook or online journal to understand more about child protection, or recognising signs of child abuse in the clinical setting. Again, this depends on their own initiative to find the appropriate materials to enhance their knowledge and skills. Else the only opportunity available is though hands on experience from the clinical setting or direct teaching and supervision by their superior. This could be an advantage or disadvantage because it depends on how much knowledge transfer had transpired within the setting, some might gain more while others not so much, resulting in knowledge gap amongst healthcare professionals.

\section{Subtheme 3: Content of training}

According to the professional healthcare workers interviewed, the content of the current training included basic introduction on child abuse (definition, signs and symptoms), the procedure needed to be done in dealing with child abuse, the policies, and others. As stated above, paramedics (staff nurses and medical assistants) are in charge of supporting and assisting medical officers in handling child abuse cases. They are also in charge of collecting evidence for processing and to 
produce in court and to the police. Hence, most training for these paramedics are only limited to understanding procedures in collecting and processing specimens for the purpose of producing evidence in court, if the child abuse case would proceed to the court.

\section{Key Theme 3: Risk perception}

Clarity in distinguishing between an abuse and an accident case were also noted as one of issues among the healthcare workers. One of the participants mentioned that an intracranial bleeding injury did not necessarily mean that the child had been abused. It could be an accident whereby the caretaker might accidentally shook the baby in order to make him or her to sleep but instead, due to lack of experience and knowledge of a proper childcare, causes the baby to suffer intracranial bleeding from the shaking. These findings suggest there is a real lack of awareness within hospitals as to what defines neglect and abuse, and confirms existence of definite grey area that needs to be addressed so that all findings of child neglect and abuse can be defined, standardised and identified.

Appropriate reaction time to act on abuses other than physical, especially neglect was also discovered as illustrated by one medical officer,

There are a few cases like [clears throat] where we take a full history is actually immunisation is not complete? Because parents don't believe in those things? Which I think it should be as neglected as other cases as well? So those are the cases that I think some of the medical officers don't think that they need to pursue.

One doctor took this matter into light where she said that Malaysia is a country where multiple cultures and beliefs are allowed to be practiced freely. The diversity of cultural practices and belief against immunisation had led the medical officers in conundrum on choosing between reporting such practice which would interfere with the human right of the family versus becoming an accessory to neglect for denying the right of the child to access healthcare services (such as treatment). All of these are the effect of not having a high suspicious index in order to pick up children coming in with presentation that raises certain "red flags" due to lack of knowledge in understanding in depth what is child abuse.

\section{Key Theme 4: Awareness of detection tools}

When being asked about the presence of child abuse detection tools in the hospital, most of the healthcare workers interviewed were not aware that such tools are in existence. This is confirmed by one of the medical officers,

To tell the truth, in Emergency, there is no fast and hard guideline for certain areas, especially...areas, including children.

Despite absence of tools to identify or detect child neglect or abuse, there are checklists available for use at the participating hospitals. Most of the checklists that are currently present are those that provide guidelines regarding the procedure (Standard Operating Procedure for One Stop Crisis Centre) of dealing with child abuse such as taking samples, making a report, and a clerking sheet which is used mainly by medical officers and specialists whereas case report is used by nurses. Again, this Standard Operating Procedure (SOP) will only come into play when the child has already been suspected of being abused by the attending medical officer before full examination to determine the suspicion is being carried out in the One Stop Crisis Centre (OSCC).

Majority of participants provided supportive agreement and attitude with the development of screening tool for child abuse as it can be used as a standardise tool to screen all children attending the department regardless of their presenting 
symptoms. The standardised checklist/ screening tool would assist healthcare worker in screening in or out symptoms which would benefit them a lot especially for new staffs in the frontline such as medical assistant as the assessment is more objective than perceptive in nature.

This idea was fully supported by one medical assistant during the interview,

I can look for, more objective lah meaning you have all the documentation and everything lah based on the criteria then push lah (for case refer to OSCC). I believe eh the first person to see the patient can affect the final outcome...

This is of great importance as it will potentially save a life of a child. However, one medical officer argued that although a screening tool can be beneficial, it could also be a problematic,

Sometimes, the way the assessor understands or perceives from the screening tool would probably make the case...it's probably just a normal kid and sometimes uhmm it turns out to be the suspected abuse because the way they perceive.

From this statement, the medical officer emphasised that a screening tool might also lead to an over-estimation of a case, possibly generating more false positive situations where more children are screened for being potentially at risk of abuse resulting in unnecessary conflict and pressure for the child and their family. Not to mention the amount of added work needed to be done by the staff of the department, which would include thorough examination, collection of samples for tests and consultation with members of the Suspected Child Abuse and Neglect (SCAN) team. Therefore, the content of the tools must be properly tailored.

Most healthcare workers mentioned the importance of looking at physical signs such as bruises, atypical fractures, foreign cutaneous marks, body weight and also radiological images that could suggest a possible atypical injury when solicited on what should the checklist contains. The inconsistency of the history and the child's injury was also suggested as one of the most important components of the detection tool. Some of the healthcare workers also recommended including family history because certain family situations could lead to child abuse.

One medical officer gave an example of the family history needed,

For example, the...definitely the income... of the family members. And then the errr...involvement of the family members with certain... drugs.

Other examples of family history that should be taken into consideration were added by another medical officer,

Sometimes we tend not to ask
about the father...urmm, about the
father, the father is alcoholic, any...
pernah masuk penjara ke? Has been
jailed before?

Immunisation history, close-ended questions to capture derogatory remarks or key words were recommended by some healthcare worker to be included into the screening tool to assist detection of neglect. One of the recommendations was also to customise the screening tool according to ages. Other recommendations included the number of presentations to the hospital, any recurrent infections, status of the person who bring the child in, and also the amount of the similar complaints whenever a child is presented to the hospital.

\section{DISCUSSION}

Medical healthcare professionals, being the first point of contact and front liners in the medical setting, provides an opportunity for children potentially at risk of being abused and neglect to be identified. During 
the course of the interviews, the researcher found that a two-tiered system exist within the healthcare setting, with medical practitioners on one end and paramedics (such as nurses and medical assistants) on the other (see Figure 1).

Often enough, paramedics were reluctant to raise any warning signs to the attending medical officer if he/she found that a child coming into the ED could be potentially at risk of being abused/neglected. There were contradicting statements coming from both ends whereby medical practitioners said to have encouraged paramedics to bring up warning signs of abuse to them but observed that most of the time none of the nurses or medical assistants would do so. But when nurses and medical assistants were prompted on whether they would bring it up if these warning signs occur, most of the time they said that they would say yes but in practice they would not do so. It could be because they felt that they might be overstepping their boundaries as their role is mainly to assist and provide support to attending medical officers or could be because they do not have the authority to diagnose, hence, may not have adequate knowledge to do so. The same sentiment was reported in the study done by Lazenbatt and Freeman, where they found that nurses may also feel some discomfort and anxiety from dealing with child abuse and neglect (CAN) cases and they might have fears from the consequences of their actions (3).

It could also be the fact that being paramedics in the ED, they are usually extremely busy carrying out their respective duties and tasks that are at hand, any other additional tasks would be an added burden and overwhelming for them. It should also be noted that because the standard operating procedure in the ED clearly stated what their roles and responsibilities are, any other instruction given verbally usually does not carry much weight. Anything that is not stated in the SOP, protocols or guidelines might also incur repercussions, which also added to the lack of motivation for paramedics to take on the initiative to raise the alarm in the first place. There should not be any issue or concern about raising false alarm because if all children are being screened in the first place, there is less chances of children who may fall under the radar.

In other countries such as Australia, nurses' duties to report CAN are expressed in legislation, or in occupational policy documents (4). The Australian Nursing \& Midwifery Federation (ANMF) also had issued a policy statement in 2007 which clearly stated, "It is essential for anyone

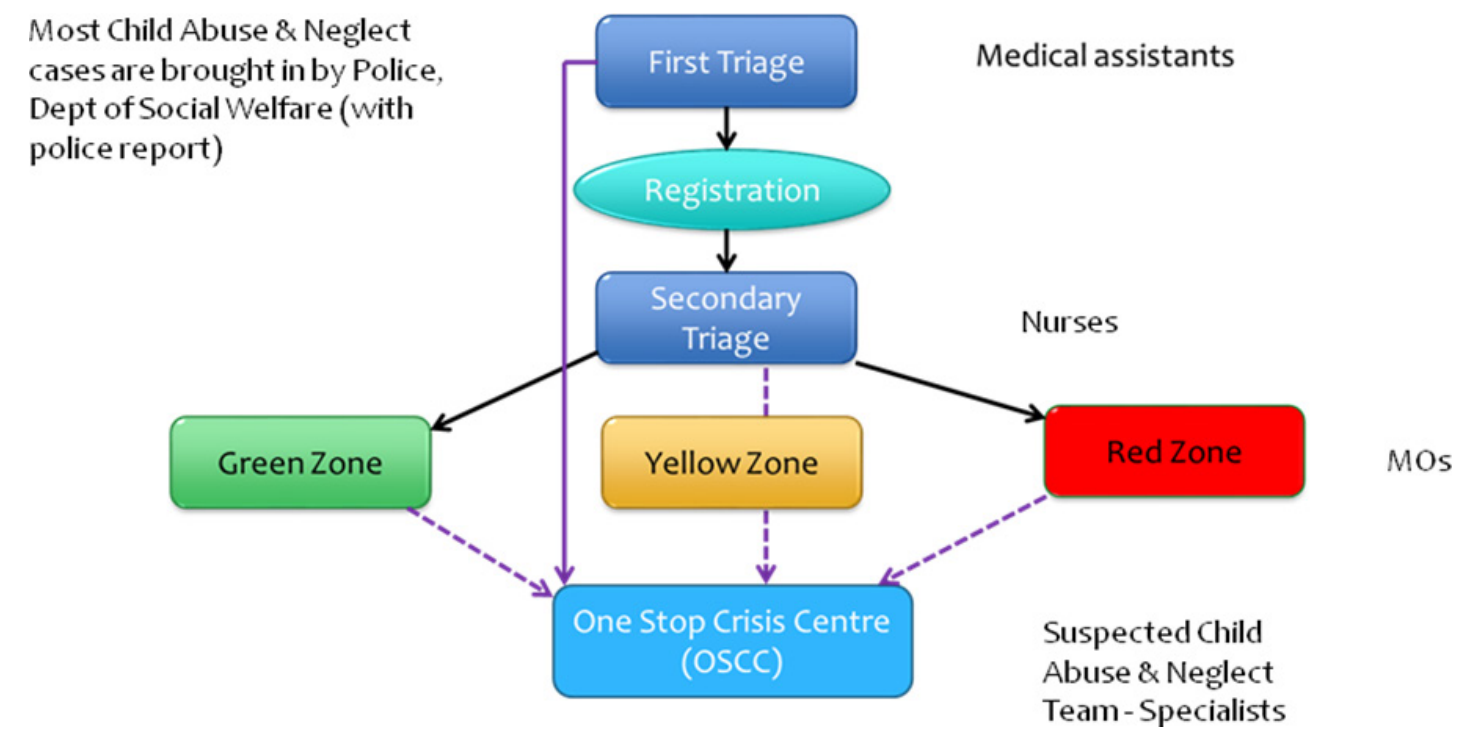

Figure 1: Flowchart of patient coming into the ED. 
working with children or young people to recognise their vulnerable status and the special protection they may require if they appear to be at risk" (5).

Similar legislation and systems are being implemented in Taiwan where the nurses have their own position in the healthcare settings and have their own voice in reporting CAN cases within their respective setting. Therefore, it is important that paramedics are also held accountable to provide more than just assistance and support in terms of services to patients (screening in the first triage and taking vital signs in the second triage). Being front liners as the initial first contact in the first and second triage, they play a very important role but are currently heavily underutilised. This should be done at all hospitals and if possible, to be included in an addendum within the current SOP. So that it can be formalised in such a way that identifying children who are potentially at risk of neglect abuse, and bring it to the attention of the person in charge are also part of their duty and not just those who are assigned in the SCAN team.

Likewise, attending medical officers carry the responsibility to identify children who are suspected of neglect or abuse, but they also need support, encouragement and empowerment on their vital role in identifying children who are at risk of neglect or abuse. This would encourage them to spend extra time on children whom they have high suspicions of potentially being abused and neglect.

\section{Knowledge Gap}

There is a gap in terms of understanding early warning signs of abuse among the healthcare professionals. Some of the points highlighted during the interview were:

\section{Inadequate training on $\mathrm{CAN} /$ child} protection;

2. No standardised training across all hospitals on CAN/child protection;
3. Different level of training provided for medical officers and paramedics;

4. Uncertainties in identifying the level of severity of neglect for diagnosis; and

5. Inability to ascertain emotional and psychological abuse masked by other presenting signs and symptoms.

Even for those who are at a senior/specialist level were still unable to confirm that throughout their career they had never missed a sign of abuse. The same medical healthcare professionals reiterate again that more specialised training is needed focusing only on CAN or child injury prevention or protection. Paramedics (nurses and medical assistants) should be included in all levels of training and emphasised on their role in identifying and reporting to their superior of any children who are potentially at risk of being abused in their respective setting. This would enable them to receive the support, encouragement and empowerment to undertake their duties in promoting awareness on CAN in the clinical setting. This is of utmost importance because nurses and medical assistants are those who will be the first person to be in contact with the patient, hence have the opportunity to screen children who are potentially at risk of abuse and direct them straight to OSCC for further investigation.

Therefore, if several warning signs have been raised in the first and second triage there is enough evidence for the attending medical officer do further examination on the child away from the suspected perpetrator(s) (e.g., parents, caregivers or any other family members). This would set precedent to inculcate the culture of screening in all children presented at the ED as opposed to screening out.

Current training available for medical healthcare professionals is based on the twotiered level. These trainings can be either part of their continuing medical education (CME) or more specific to OSCC training whereby topics related to domestic abuse 
and child abuse are taught together in the training. Or in some training child abuse and neglect is being focused on, and this depends on who leads the training. These trainings are usually organised internally by certain departments or being organised by other hospitals whereby certain medical officers, nurses and medical assistants are identified to attend those trainings.

Therefore, it is important that paramedics, medical officers and housemen are able to attend a more specialised training looking at more in-depth information of child abuse and neglect detection regardless of their position. For example, in the United Kingdom (UK), each hospital has the responsibility to provide adequate safeguarding children training. In King's College Hospital, they are responsible in providing all clinical staff with child protection awareness training. They also ensure that all staffs are given information about the importance of child safeguarding and how they can support the needs of vulnerable children and young people. They also provide dedicated help through their specialist Safeguarding Children team to identify, protect and care for those at risk. And they also work with general practitioners (GPs), social services and other professionals to ensure the needs of vulnerable children and young people are met (King's College Hospital, 2017).

Providing adequate specialised training is important but providing regular training is also critical to the development of a skilled medical healthcare professional and to achieving outcomes of safety, permanency, and well-being for children entrusted to the public health system. Having this knowledge not just assist the technical aspect of identifying children potentially at risk of abuse, but it also increases the level of awareness of such case but also increases one's level of suspicion if they do come across cases that may potentially be a CAN case hence increase the sense of obligation and responsibility of officers in the ED. And this cuts across all levels be it paramedics or medical officers or specialists and consultants.

\section{Community and Work Culture}

Organisational culture in the healthcare setting has been long discussed in the Western world, but not so much in the ASEAN region. According to the Organisational Culture Profile (OCP) instrument, performance orientation culture is shaped by the following characteristics: enthusiasm for the job, results orientation, highly organised employees and high-performance expectations (6). Supportiveness and constructive organisational culture are also needed to generate a sense of collectively and emotional attachment that develops a community focused on organisational goals. Working as a team encourages more exchange of information and because of the frequent contacts, members are aware of who possesses the knowledge to resolve specific problems (6).

But what was found and observed during this study was that nurses were less enthusiastic with their work and more dependent on the senior staff nurse when encountered with complex cases such as child abuse and neglect cases. This was noted visibly among junior nurses, who were highly dependent on community nurses who manage the OSCC when it comes to SOPs and guidelines in handling CAN cases. Because the fact that they are on rotational basis, they would not have the opportunity to undergo any OSCC training hence did not feel the need to undertake additional initiative to understand more on CAN.

Community culture also acts as a barrier for nurses to respond towards CAN cases. Several researchers found that nurses considered addressing the phenomenon of child abuse to be an ethical obligation and they adopted the role of patient advocacy to support victims (7). This is particularly true especially when they have more experience working with children and had dealt with a lot of CAN cases as it was found in this 
research. While other nurses considered CAN to be a personal and family issue in which they had no role or say hence the reluctance to highlight the matter and bring it to the attention of medical officers or attending physician (8). This is especially true within the Malaysian cultural context, which is quite similar with other Arab and eastern culture where child rearing can be it is considered socially acceptable for parents to practice physical punishments and yelling to their children as a normal part of childrearing $(9,10)$.

\section{Screening Tools for Child Abuse}

This study managed to establish that there are no screening/detection tools being used at the ED or any other clinical department in the public hospital as of current. What is currently available is Form 9, used by medical officers once a child is being diagnosed as CAN case and is brought into OSCC for further examination and investigation. Prior to that, there are no checklist or screening tools being used at the first triage or second triage at the ED.

It is also found that there is very low awareness on screening or detection tools for child abuse and being used worldwide by most of the healthcare professionals interviewed except for specialist in the ED and paediatrics. There are several wellknown screening/detection tools being used in the US, Europe and Australia such as ESCAPE Instrument, SPUTOVAMO, SPUTOVAMO-R and the most recent one SPUTOVAMO-R2 (it does not only look at the injury, but also at the interaction with parents and child) and being used widely in the ED. Tool such as ISPCAN Child Abuse Screening Tools (ICAST) is another alternative for consideration with caveat that it is widely used in community and homes rather than in the ED setting.

Having a standardised screening/detection tool would assist healthcare professionals greatly in identifying children who are potentially at risk of abuse in the ED especially at the first and second triage, where paramedics are stationed as shown by Louwers et al. in the study they conducted in 2014 (11).

\section{CONCLUSION}

There is a significant gap identified in healthcare professionals' knowledge and skills related to understanding what does CAN entails and how it is being identified and detected, particularly among medical officers who are in charge of diagnosis and making police reports of any CAN cases in the hospital. Those who had previous experience or encounters in dealing with CAN cases would have higher suspicion index compared to those who haven't.

Paramedics such as nurses and medical assistants also need to have basic knowledge and awareness on CAN despite not having the mandate to diagnose or report CAN cases. They should also be encouraged and empowered to take an active role in identifying or raising the red flag with high index of suspicions so that these children can be brought in for further examination by medical officers. Similarly, leveraging and empowering experienced staffs who had been in the system long enough would help increase raising the matter for attention to attending medical officer as they are more experienced in dealing with CAN cases, more skilful and aware compared to newer recruit in the department. Consequently, the process of conducting qualitative study amongst medical healthcare professional has some impact in creating awareness on CAN in the clinical setting.

Specialised and intensive standardised training in child protection and awareness on CAN are needed and should be made available for all healthcare professionals to attend and improve their knowledge and understanding on the differentiation between accidental and non-accidental injuries as it presents at the ED. These training should be on-going with reviews and updates on the latest information on detection of CAN cases. 
Awareness on importance and severity of CAN should be made onto parents to inculcate the right interventive behaviour when faced with suspected child abuse case. Reluctance of attending medical officer in making report even with evidence (diagnosis) presence due to fear of making false report should be a strong reason to encourage parents to make report themselves. This should also trigger similar reflection with the medical officer to have more confident in making diagnosis and consulting their superior to remove barrier of reporting faced due to lack of knowledge and experience.

Front-line staffs (the medical healthcare workers) should be mandated to carry the responsibility of "protector" by higher authority to help identify case at the onset and remove the children from potential harmful and hostile environment. There is a need for enhancement and strengthened healthcare system to ensure necessary strategies are in place such as availability of screening tool and checklist to assist healthcare professional to make the diagnosis and speed up the process of identification and reporting more efficiently. About one in three children will suffer subsequent abuse showing the importance of early diagnosis of CAN. This study exemplified the need to implement such standardised tool in the ED in all hospitals which is scalable due to its standardised form. There are several tools that can be used for pilot implementation (e.g. ESCAPE form, SPUTOVAMO-R), therefore it is envisaged that these can be done as a post-doc research study in one of the hospitals.

The current available electronic database can be enhanced to assist medical professionals by linking up services with the Child Registry under the DSW and communicate with other agencies to enhance cooperation at the inter-agency level to ensure smooth and efficiency reporting and transition of the child from the clinical setting to a safer home/ environment under the protection of the DSW officers. Medical healthcare professionals, being at the forefront has to play their vital role in ensuring that every child that passes through the ED everyday would be dealt with in mind that they are all potentially at risk of being abuse and neglect in order to potentially save one life. We as part of society and community also has our role to play by creating awareness towards CAN and ensuring that every child in our community is safe and protected at all times, then maybe we would see reduction in number of CAN cases in future.

\section{ACKNOWLEDGEMENTS}

The authors would like to thank the Director General of Health Malaysia for the permission to publish this article and would like to acknowledge Head of Departments, Dr. Sabariah Jamaluddin of Sg. Buloh Hospital, Datuk Dr. Alwi Abdul Rahman of Selayang Hospital, and Dr. Rohaizah Borhan of Serdang Hospital. Authors are also grateful to Dr. Hazlina Mohd Noh and Dr. Noor Azyati Mohd Yusooff for their kind assistance during data collection at their respective hospitals.

\section{REFERENCES}

1. Peden MM. World report on child injury prevention. Geneva: World Health Organization; 2008, 233 p.

2. Roach JO. Injuries kill over 20,000 children a year in developed countries. BMJ. 2001; 322(7282):317. https://doi.org/10.1136/bmj. 322.7282 .317

3. Lazenbatt A, Freeman R. Recognizing and reporting child physical abuse: a survey of primary healthcare professionals. J Adv Nurs. 2006;56(3):227-36. https://doi.org/ $10.1111 / j .1365-2648.2006 .04030 . x$ 
4. Fraser JA, Mathews B, Walsh K, Chen L, Dunne M. Factors influencing child abuse and neglect recognition and reporting by nurses: a multivariate analysis. Int J Nurs Stud. 2010;47(2):146-53. https://doi.org/ 10.1016/j.ijnurstu.2009.05.015

5. The Australian Nursing \& Midwifery Federation. Position Statement on child abuse and neglect [Internet]. Accessed in July 2007. Available from: https://www. google $\cdot \operatorname{com} / \mathrm{url}$ ? sa $=\mathrm{t} \& \mathrm{rct}=\mathrm{j} \& \mathrm{q}=\& \mathrm{esrc}$ $=\mathrm{s} \&$ source $=$ web $\& \mathrm{~cd}=2 \& \mathrm{cad}=$ rja\&uact $=8$ \&sqi $=2 \& v e d=0$ ahUKEwiDsJC $57 \mathrm{~N}_{-}$ UAhXGOo8KHY5sDTUQFggpMAE\&url $=$ http $\% 3 \mathrm{~A} \% 2 \mathrm{~F} \% 2 \mathrm{Fanmf}$.org. au $\% 2 \mathrm{~F}$ documents\%2Fpolicies\%2FPS_Child_abuse _and_neglect.pdf\&usg =AFQjCNHWc JIWIREsBNyg4uehM0CiyifaDw

6. Zachariadou T, Zannetos S, Pavlakis A. Organizational culture in the primary healthcare setting of Cyprus. BMC Health Serv Res. 2013;13:112. https://doi. org/10.1186/1472-6963-13-112

7. Bannon MJ, Carter YH. Paediatricians and child protection: the need for effective education and training. Arch Dis Child. 2003;88(7):560-2. https://doi.org/10.1136/ adc. 88.7 .560
8. Alrimawi I, Saifan AR, AbuRuz M. Barriers to Child Abuse Identification and Reporting. J Appl Sci. 2014;14:2793-803. https://doi. org/10.3923/jas.2014.2793.2803

9. Chavis A, Hudnut-Beumler J, Webb MW, Neely JA, Bickman L, Dietrich MS, Scholer SJ. A brief intervention affects parents' attitudes toward using less physical punishment. Child Abuse Negl. 2013;37(12):1192-201. https://doi. org/10.1016/j.chiabu.2013.06.003

10. Elbedour S, Abu-Bader S, Onwuegbuzie AJ, Abu-Rabia A, El-Aassam S. The scope of sexual, physical, and psychological abuse in a Bedouin-Arab community of female adolescents: the interplay of racism, urbanization, polygamy, family honor, and the social marginalization of women. Child Abuse Negl. 2006;30(3):215-29. https://doi. org/10.1016/j.chiabu.2005.10.010

11. Louwers ECFM, Korfage IJ, Affourtit MJ, Ruige M, van den Elzen APM, de Koning HJ, Moll HA. Accuracy of a screening instrument to identify potential child abuse in emergency departments. Child Abuse Negl. 2014;38(7):1275-81. 